\title{
Experiência com o uso da Ferramenta Scratch na Terapia de Pacientes Psiquiátricos
}

\author{
Lucas Borges Leal ${ }^{1}$, Keylla Maria de Sá Urtiga Aita², Aline Montenegro Leal \\ Silva ${ }^{2}$ \\ ${ }^{1}$ Curso de Licenciatura em Computação - Universidade Federal do Piauí (UFPI) - \\ Universidade Aberta do Brasil (UAB) - Polo de Inhuma \\ 64049-550 - Teresina - PI- Brasil \\ ${ }^{2}$ Centro de Educação Aberta e à Distância - Universidade Federal do Piauí (UFPI) - \\ Rua Olavo Bilac, 1148, 64001-280, Teresina - PI- Brasil \\ lucasboorges13egmail.com, keyllaurtigaeufpi.edu.br, \\ alineleal50yahoo.com.br
}

\begin{abstract}
This article aims to present experiences of users with mental disorders through the Scratch tool. The users make use of the services of the Psychosocial Attention Center (PAC) that aims to promote social reintegration through various modalities of psychic treatments, among which are: therapeutic activities and treatment by medication. The use of the Scratch tool was mediated by the neuropsychologist who assisted the patients in performing the play activities. The Scratch tool serves as an exercise for the mind and for motor coordination. The objective of this work is to promote the interference of digital resources in the space of therapies, as a way of digitally including this portion of the population.
\end{abstract}

Resumo: O presente artigo destina-se a apresentar vivências de usuários com transtornos mentais por meio da ferramenta Scratch. Os usuários fazem uso dos serviços do Centro de Atenção Psicossocial (CAPS) que objetiva promover a reinserção social por meio de várias modalidades de tratamentos psíquicos, dentre os quais se destacam: atividades terapêuticas e o tratamento por medicação. $O$ uso da ferramenta Scratch foi mediado por neuropsicopedagogo que auxiliou os pacientes na realização das atividades lúdicas. A ferramenta Scratch serve de exercício para a mente e para a coordenação motora. O objetivo do trabalho é promover a interferência de recursos digitais no espaço das terapias, como forma de incluir digitalmente essa parcela da população.

\section{Introdução}

Após a reforma psiquiátrica ter se consolidado como política oficial do governo federal, assuntos relacionados à saúde mental tem sido objeto de discussão constante nas pautas governamentistas. De acordo com Ribeiro e Dias (2011), o processo de inovação da psiquiatria se desenvolveu após intervenções das entidades sociais (Federal, Estadual e municipal), que se valeram de movimentos sociais para concretizar a transição de um modelo de assistência centrado em manicômios para um modelo de atenção comunitário e humanizado, mediados pelos Centros de Atenção Psicossocial (CAPS).

Segundo Acioly (2009), um dos pontos positivos da reforma psiquiátrica foi a implantação de serviços de atendimento extra hospitalares, como os CAPS, Centro de Atenção Diária (CAD), Hospitais Dias (HDs) e outros. Porém a época não foi marcada apenas por glórias. Os primeiros tratamentos a esses pacientes não eram tachados 
VIII Congresso Brasileiro de Informática na Educação (CBIE 2019)

Anais do XXV Workshop de Informática na Escola (WIE 2019)

humanamente, os primeiros hospitais foram construídos, não para oferecer o tratamento, mas para afastá-los do convívio da sociedade, e por séculos, essas pessoas foram completamente abandonadas em casas de isolamento, não sendo, portanto, detentoras de direitos básicos de cidadania.

Com o avanço da tecnologia, diversas áreas sociais e da saúde sofreram mutações, e com a saúde mental não foi diferente. Após a implantação desse novo modelo de tratamento, a prática do "cuidar do deficiente mental" também incluiu a utilização de recursos digitais, o que tornou as terapias mais interativas e mais lúdicas. A presença da tecnologia tende a trazer uma socialização imensurável aos Centros de Apoio Psicossocial quebrando assim, a tensão dos manicômios, teoria na qual se firma nas palavras de Basaglia (1982): "Quando dizemos não ao manicômio, estamos dizendo não a miséria do mundo e nos unimos a todas as pessoas que lutam por uma situação de emancipação".

Neste contexto, apresentamos nesse trabalho as experiências obtidas com o uso da ferramenta Scratch no ambiente terapêutico dos pacientes psiquiátricos junto ao neuropsicopedagogo. O Scratch é uma linguagem de programação desenvolvida pelo Instituto Tecnológico de Massachussetts para a criação de estórias interativas, jogos e animações que são feitos a partir de comandos montados e seguindo uma sequência lógica. Durante o período de aplicação dessa ferramenta, que foi por um intervalo de tempo de três semanas consecutivas, sendo utilizada três dias por semana, foram acompanhados seis pacientes internos do CAPS. A aplicação se deu por meio de jogos e animações disponibilizados pelo programa e cada exercício trabalhou as necessidades especificas de cada paciente. A finalidade desse experimento foi trabalhar as desenvolturas cognitivas dos mesmos, sendo elas, coordenação motora, noções de lateralidade, visão seletiva e a interatividade.

\title{
2. Inclusão digital e o uso de tecnologias na terapia da saúde mental
}

O termo "inclusão digital" pode compreender uma série de significados abrangidos em diversas áreas, mas, em linhas gerais, entende-se como uma forma de apoio aos cidadãos na perspectiva de inserção na sociedade contemporânea. Graham (2004) afirma ainda:

\begin{abstract}
"As Tecnologias de Informação e Comunicação (TICs) podem tanto proibir como aumentar a liberdade e a mobilidade, mostrando exemplos onde softwares dão prioridade ao acesso de uns em detrimento de outros, dificultando ou facilitando a inclusão digital espontânea. São softwares que priorizam socialmente o tráfego na internet, nas esperas das chamadas nos Call Centres, nas autoestradas e na midiatização da geografia social através de sistemas geodemográficos. Softwares invisiveis e automáticos que passam despercebidos, mas que, segundo o autor, são um elemento crítico da exclusão digital e que perpetuam as desigualdades nas cidades contemporâneas."
\end{abstract}

Existe todo um arsenal de recursos digitais promotores da inclusão de deficientes mentais que vão desde o mais acessível ao mais técnico. Segundo Nunes et al. (1998):

“[...] adaptações especiais como tela sensível ao toque ou ao sopro, detector de ruídos, mouse alavancado à parte do corpo que possui movimento voluntário e varredura automática de itens em velocidade ajustável, permitem seu uso por virtualmente todo portador de paralisia cerebral, qualquer que seja o grau de seu comprometimento motor."

De acordo com Fortuna e Oliveira (2011), a reforma psiquiátrica não supriu todas as necessidades, há uma demanda por informações acerca das terapias substitutivas em saúde mental que valorizem a prática no Brasil. Embora algumas experiências de oficinas midiáticas tenham sido relatadas, as informações a este respeito - onde ocorrem, 
metodologias e resultados - encontram-se dispersas na literatura. Ainda por meio do estudo de Fortuna e Oliveira (2011), foi feita uma identificação preliminar das oficinas midiáticas comunicacionais como atividade psicossocial nos serviços de saúde mental no Brasil, onde foi possível localizar um número significativo de experiências de práticas terapêuticas comunicacionais em serviços de saúde mental através de oficinas de rádio, fotografia, web rádio, TV, construção de jornal, sites e produção de vídeos.

Com base nas abordagens conceituais sobre o uso de tecnologias na terapia da saúde mental, é possível notar pontos de convergência nos posicionamentos dos autores que focaram esse tema. Os conceitos dos autores citados acima estão diretamente relacionados à proposta desta pesquisa pelo fato dos mesmos tratarem a inclusão social dos usuários do tratamento da saúde mental com o apoio das tecnologias. Por exemplo, Graham (2004) não se intimida ao relatar softwares como facilitadores no tratamento da saúde mental. Fortuna e Oliveira (2011) focam na utilização direta de recursos digitais como auxiliadores do tratamento.

A pesquisadora Oliveira (2016) em sua tese de doutorado realizou um estudo bibliométrico, em que buscou analisar na literatura a aplicação de instrumentos tecnológicos para o auxílio em diagnóstico e tomada de decisões médicas em saúde mental, mais especificamente sobre depressão, de modo a justificar a importância e a necessidade de aplicativos e softwares que possam ser usados para melhorar o atendimento de portadores de transtornos depressivos, de acordo com a tabela 1.

Tabela 1. Estudos sobre instrumentos tecnológicos aplicados à saúde mental

\begin{tabular}{|c|c|c|c|c|}
\hline \multirow[b]{2}{*}{ TÍTULOS } & \multicolumn{4}{|c|}{ CATEGORIAS } \\
\hline & $\begin{array}{l}\text { Questionário } \\
\text { Digital }\end{array}$ & Aplicativo & $\begin{array}{l}\text { Atenção } \\
\text { Primária à } \\
\text { Saúde }\end{array}$ & $\begin{array}{c}\text { Clínicas e } \\
\text { Ambulatórios }\end{array}$ \\
\hline 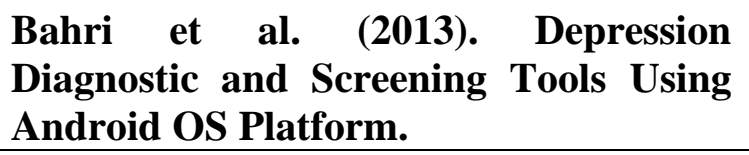 & & $X$ & & \\
\hline $\begin{array}{l}\text { Borycki \& Keay (2010). Methods to } \\
\text { Assess the Safety of Health Information } \\
\text { Systems. }\end{array}$ & & $\mathrm{X}$ & & \\
\hline $\begin{array}{l}\text { Carey et al. (2014). Acuracy of general } \\
\text { practitioner unassisted detection of } \\
\text { depression. }\end{array}$ & & $X$ & & $\mathrm{X}$ \\
\hline $\begin{array}{l}\text { Chattopadhyay (2014). A neuro-fuzzy } \\
\text { approach for the diagnosis of } \\
\text { depression. }\end{array}$ & & $\mathrm{X}$ & $X$ & \\
\hline $\begin{array}{l}\text { Cook et al. (2006). Electronic source } \\
\text { materials in clinical research: } \\
\text { acceptability and validity symptom } \\
\text { selfrating in major depressive disorder. }\end{array}$ & $X$ & & & $X$ \\
\hline $\begin{array}{l}\text { Drozd et al. (2015). An Internet-Based } \\
\text { Intervention (Mamma Mia) for } \\
\text { Postpartum Depression: Mapping the } \\
\text { Development from Theory to Practice. }\end{array}$ & $X$ & & & $\mathrm{X}$ \\
\hline
\end{tabular}


VIII Congresso Brasileiro de Informática na Educação (CBIE 2019)

Anais do XXV Workshop de Informática na Escola (WIE 2019)

\section{Gardner et al. (2004). Computerized adaptive measurement of depression: A simulation study.}

No que tange ao uso do Scratch, software alvo do presente experimento, tem-se que sua versatilidade o faz ferramenta com múltiplas utilidades. A facilidade no manuseio juntamente com os vários recursos (sonoros e visuais) permitem que este software seja direcionado a todo o público. $\mathrm{O}$ trabalho com o Scratch proporciona aos usuários a oportunidade de desenvolverem diferentes habilidades, auxiliando o processo de aprendizagem e facilitando a aquisição de competências para resolução de problemas. A tabela 2 mostra as experiências de alguns autores que utilizaram a ferramenta.

Tabela 2. Estudos encontrados sobre a ferramenta Scratch

\begin{tabular}{|c|c|}
\hline $\begin{array}{l}\text { Bastos, Borges } \\
\text { e Abreu } \\
(\mathbf{2 0 1 0 )}\end{array}$ & $\begin{array}{l}\text { Relatam que o Scratch é uma ferramenta que pode ser usada para } \\
\text { auxiliar o usuário no processo de aprendizagem, tornando a } \\
\text { tecnologia um instrumento do seu desenvolvimento pessoal. }\end{array}$ \\
\hline $\begin{array}{l}\text { Escribano e } \\
\text { Montoya } \\
(2012)\end{array}$ & $\begin{array}{l}\text { Enfatizam que o Scratch implica a realização de atividade, } \\
\text { comunicação, intercâmbio de ideias, planificação, superação de } \\
\text { erros e definição de estratégias para a resolução de problemas. }\end{array}$ \\
\hline $\begin{array}{l}\text { Franzoia et al. } \\
\qquad(2018)\end{array}$ & $\begin{array}{l}\text { Apresentam a influência do Scratch no desenvolvimento do } \\
\text { Pensamento Computacional durante a criação de Objetos Digitais } \\
\text { de Aprendizagem em Física-Ondulatória. Ao longo do trabalho } \\
\text { avaliou-se a hipótese de que a produção de objetos digitais de } \\
\text { aprendizagem utilizando o Scratch pode gerar aprendizagem } \\
\text { significativa. }\end{array}$ \\
\hline $\begin{array}{l}\text { Marques } \\
\text { (2009) }\end{array}$ & $\begin{array}{l}\text { Aborda o Scratch como facilitador da aquisição de competências } \\
\text { de resolução de problemas, de concepção de planos com } \\
\text { raciocínio lógico, de reconhecimento e supressão de erros, de } \\
\text { desenvolvimento de ideias (desde a criação até a execução do } \\
\text { projeto), de concentração e de persistência. O Scratch é um } \\
\text { exemplo de um micromundo, pois disponibiliza uma série de } \\
\text { ações simples que possibilita a imersão e exploração acessível na } \\
\text { estruturação de tarefas significativas, que ajuda na compreensão } \\
\text { de ideias e princípios preponderantes de diversas áreas do } \\
\text { conhecimento. }\end{array}$ \\
\hline $\begin{array}{l}\text { Pinto, F. } \\
\text { (2015) }\end{array}$ & $\begin{array}{l}\text { Recomenda o uso do Scratch para promover a fluência digital, } \\
\text { enfatizando que ser digitalmente fluente envolve saber como usar } \\
\text { as ferramentas tecnológicas ao mesmo tempo que se consegue } \\
\text { construir algo com significado. }\end{array}$ \\
\hline $\begin{array}{l}\text { Ribeiro, S., } \\
\text { Melo, A. } \\
\quad(2017)\end{array}$ & $\begin{array}{l}\text { Apresentam uma alternativa de integração entre Interação } \\
\text { Humano-Computador e Engenharia de Software no } \\
\text { desenvolvimento de tecnologia para/com/por crianças, de forma } \\
\text { sistematizada, com o apoio do ambiente Scratch. }\end{array}$ \\
\hline
\end{tabular}


VIII Congresso Brasileiro de Informática na Educação (CBIE 2019)

Anais do XXV Workshop de Informática na Escola (WIE 2019)

\section{Método}

O desenvolvimento dessa pesquisa percorreu 6 fases (Figura 1). Inicialmente foi realizado a revisão bibliográfica sobre o tema estabelecido, em seguida um estudo da ferramenta Scratch com a finalidade de aplicá-la no Centro de Atenção Psicossocial (CAPS) do município de Inhuma (Piauí). Focando no apoio terapêutico ao tratamento dos intensivos, o estudo da ferramenta se deu por um período de dez dias consecutivos para apropriação do conhecimento necessário para a sua aplicação em campo, almejando melhorias nas desenvolturas cognitivas desses pacientes, tais como: coordenação motora, noções de lateralidade, nível de iteratividade e visão seletiva. Todo esse estudo fundamentou-se numa perspectiva qualitativa e interpretativa, em que cada paciente, foi trabalhado, primordialmente, sua maior necessidade, seguido das demais atividades.

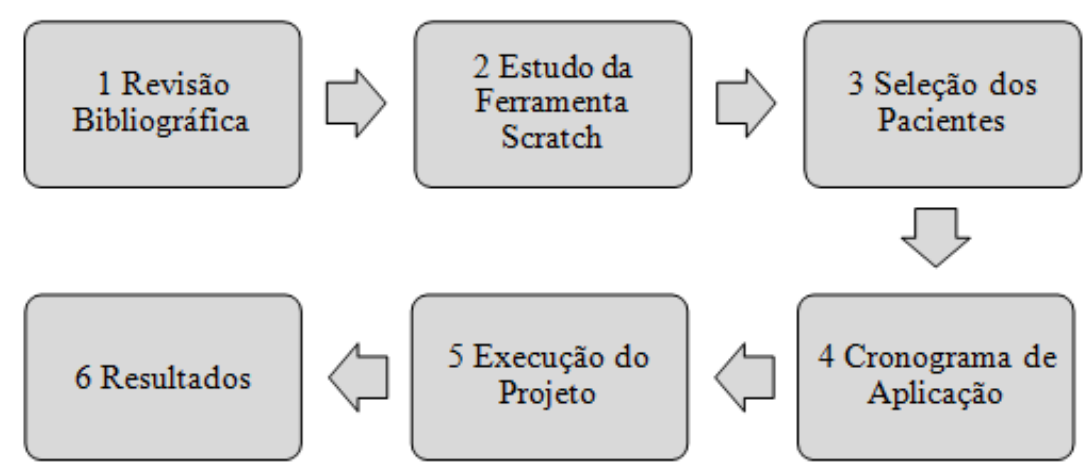

Figura 1: fluxograma da metodologia. Fonte: o autor, 2018

Após a fase de estudos partiu-se para a seleção dos pacientes, cujo requisito era a escolha dos que tinham condições de interação com a ferramenta, bem como capacidade de receber e executar instruções sobre o uso do software. Nesta seleção, constatou-se que apenas seis, dos vinte intensivos, estavam habilitados à aplicação da terapia computacional. Posteriormente foi ajustado um cronograma onde se adequou o horário de aplicação prática da terapia de acordo com a Classificação Internacional de Doenças (CID) e disponibilidade de cada paciente. $\mathrm{O}$ cronograma foi estabelecido da seguinte maneira: a aplicação prática aconteceu três dias por semana (Terça, Quarta e Quinta) por três semanas consecutivas, sendo trabalhados dois pacientes por dia com uma duração de trinta minutos cada sessão.

Para a execução da terapia, penúltima fase do experimento, foi utilizada uma espécie de jogo desenvolvido no Scratch (Figura 2). Esse artifício foi desenvolvido da seguinte maneira: criado na plataforma do aplicativo, o labirinto foi contemplado com um percurso extenso e possui várias ramificações, as quais permitem aos usuários trabalhar o controle do próprio corpo, ou seja, coordenação motora e noções de lateralidade. Dentro das ramificações existem objetos que necessitam ser alcançados pelo sujeito virtual comandado pelos usuários. Essa técnica permite trabalhar a percepção visual, já que necessita de uma atenção especial para alcançar os objetos. 
VIII Congresso Brasileiro de Informática na Educação (CBIE 2019)

Anais do XXV Workshop de Informática na Escola (WIE 2019)

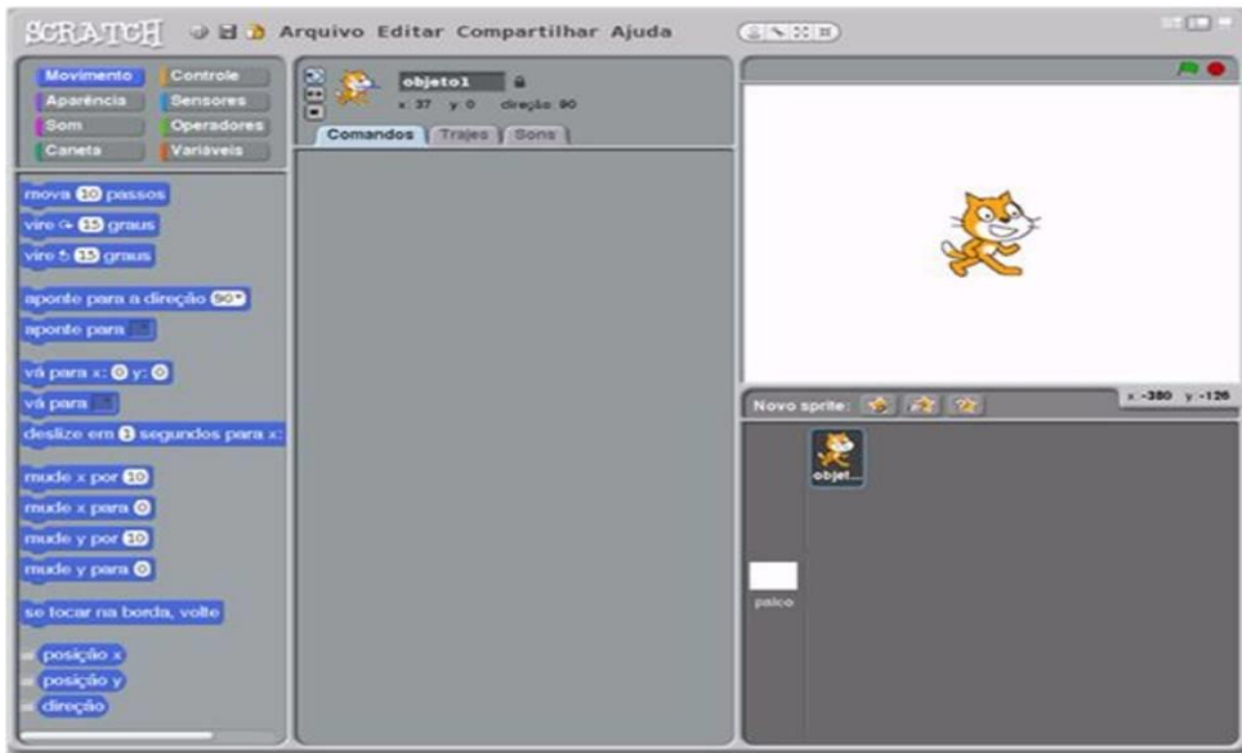

Figura 2: Tela de início do Scratch. Fonte: Autoria própria, 2018

O experimento foi realizado com apenas um paciente por sessão e a interação ocorreu da seguinte forma: o usuário delegou comandos a um sujeito virtual (gato), o qual partiu de um ponto inicial e trilhou pelo labirinto sem tocar as paredes até chegar ao destino final, finalizando automaticamente a sessão (Figura 3). As instruções do software para este experimento sugerem que o gato deve entrar em todas as ramificações do labirinto para tocar nos objetos que ali estão, sendo seis objetos no total. Outra instrução é não deixar o gato tocar nas paredes, caso contrário, o mesmo interrompia o percurso e parava no local, necessitando assim de um comando oposto ao sentido que o mesmo percorria.

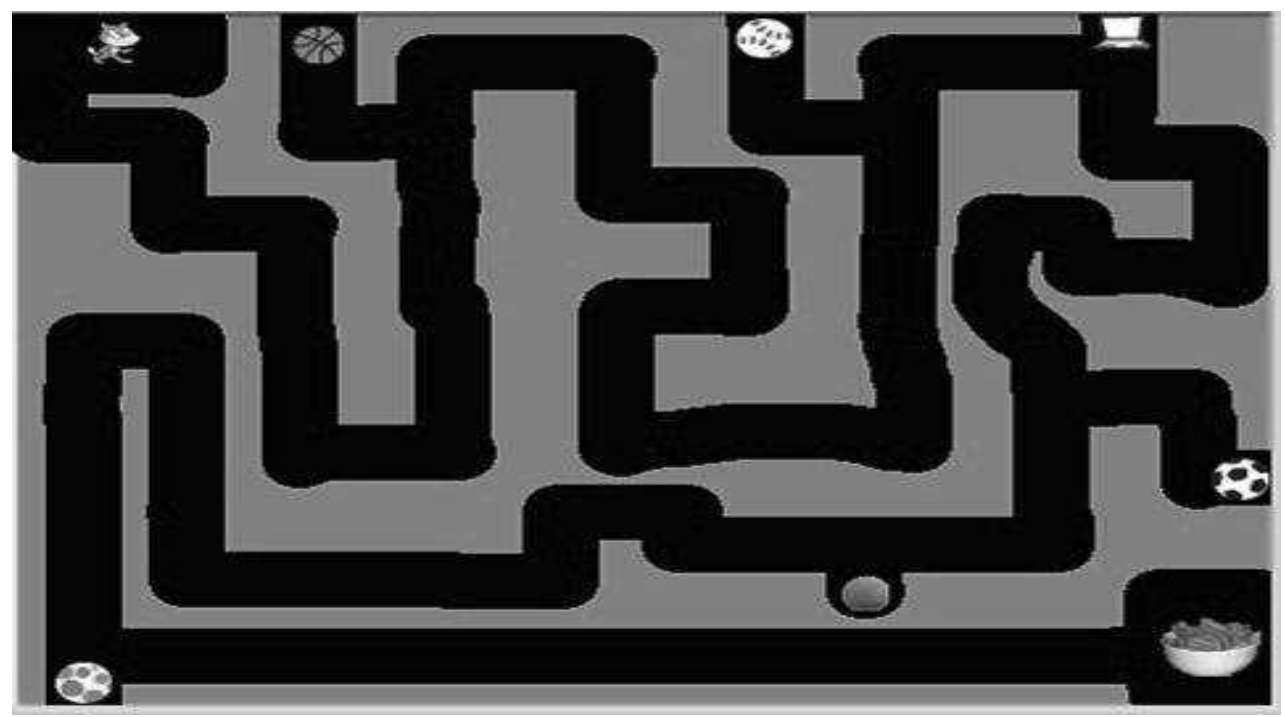

Figura 3: Tela do jogo utilizado. Fonte: Autoria própria, 2018

Ao fim da experiência foi utilizado, como instrumento de fechamento do experimento, um pequeno questionário junto aos pacientes, ainda assistidos pelo neuropsicopedagogo que buscou avaliar a assistência dada a eles, assim como as experiências vividas pelos mesmos durante as sessões. 
VIII Congresso Brasileiro de Informática na Educação (CBIE 2019)

Anais do XXV Workshop de Informática na Escola (WIE 2019)

A aplicação prática do software tem a finalidade de atender as demandas supracitadas, sendo as atividades divididas em três etapas: a primeira etapa consistiu em detectar a maior dificuldade na utilização do software; na segunda etapa trabalhou-se a superação da dificuldade encontrada; na terceira e última etapa estimulou-se a prática das demais atividades.

Todo o experimento foi acompanhado pelo profissional da instituição, o neuropsicopedagogo Adaljerry Ferreira de Sousa, que também mediou a aplicação e determinou até que ponto poderia ser trabalhado as necessidades dos usuários, isso para que o uso da ferramenta não fosse aplicado excessivamente para não ocorrer alterações inesperadas no quadro psíquico dos pacientes.

Os resultados apresentados na sessão seguinte foram baseados nas observações do neuropsicopedagogo juntamente com as impressões subjetivas dos usuários conseguidas através de uma sessão de conversa mediada por questionamentos gravados.

\section{Resultados e discussões}

A finalidade desse experimento com a ferramenta Scratch foi trabalhar as desenvolturas cognitivas dos pacientes, sendo elas, coordenação motora, noções de lateralidade, visão seletiva e a interatividade, como mostra a Figura 4. De início houve uma resistência por parte dos pacientes selecionados para realizar a aplicação, mas já era esperado pelo fato de tratar-se de uma atividade nova e completamente diferente das que estavam habituados a praticarem.

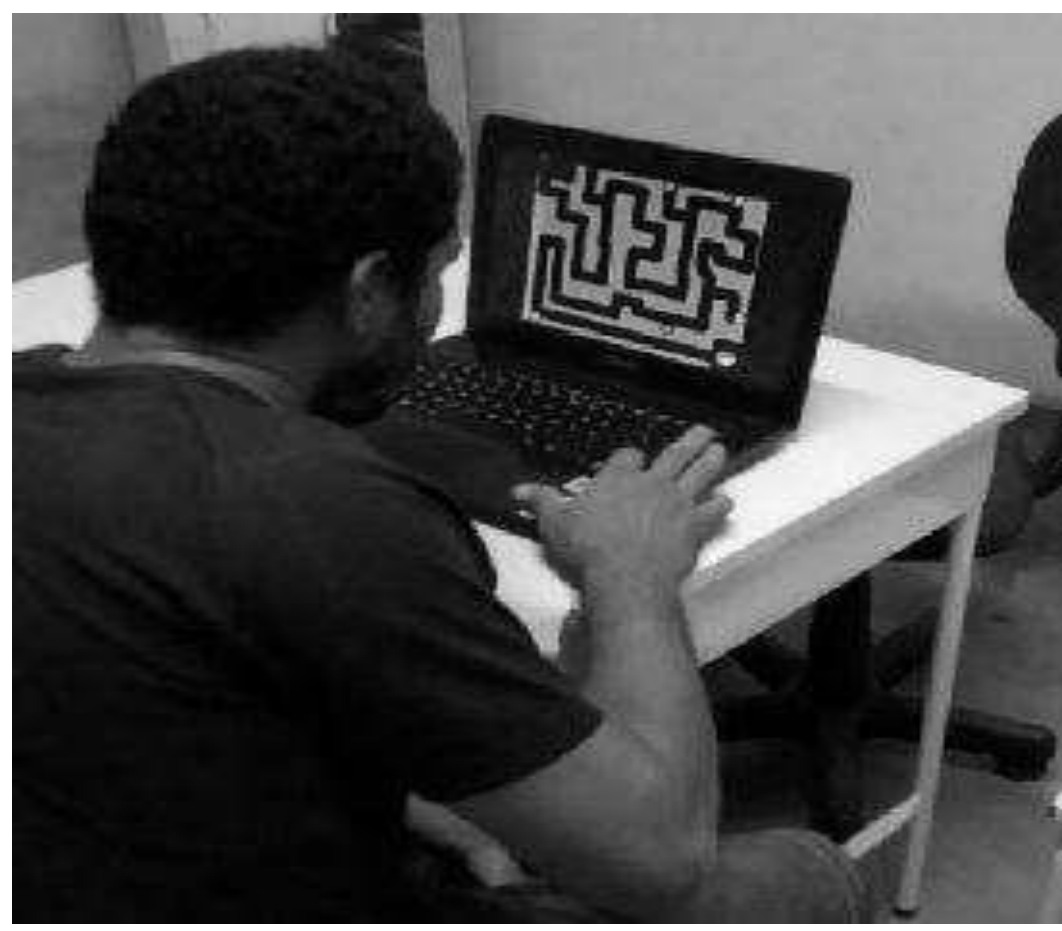

Figura 4: Aplicação prática. Fonte: Autoria própria, 2018

Ao final do experimento o Neuropsicopedagogo da Instituição, Adaljerry Ferreira de Sousa, aferiu o que segue:

"Com base em análises da utilização do aplicativo Scratch em atividades com intensivos no Centro de Atenção Psicossocial - CAPS, Inhuma (Piauí), percebeu-se que os objetivos do referido aplicativo aderem às propostas de atendimentos terapêuticos buscando uma aproximação da utilidade prática com as necessidades 
VIII Congresso Brasileiro de Informática na Educação (CBIE 2019)

Anais do XXV Workshop de Informática na Escola (WIE 2019)

delimitadas pela Classificação Internacional de Doenças (CID) de cada paciente. Com o uso dessa ferramenta pôde-se observar um trabalho intenso da coordenação motora fina que, ao longo de outras sessões de aplicação coordenadas, poderia culminar com um aprimoramento desta função, bem como do desenvolvimento psicomotor, cognitivo e de lateralidade. Com isto acredita-se que este tipo de terapia poderia amenizar episódios de ansiedade, pois utiliza ferramentas inovadoras como terapias complementares ao tratamento medicamentoso que os pacientes são submetidos."

Como atividade final foi feita uma enquete com os pacientes, na forma de uma conversa informal, a fim de obter e analisar as impressões pessoais subjetivas de como o experimento interferiu na zona de conforto deles. Porém, quase todos os envolvidos se recusaram a debater o assunto, e devido às limitações conhecidas, optou-se por não pressioná-los sob risco de alteração em seus quadros psíquicos. Apenas um deles se habilitou a falar sobre a aplicação do Scratch e revelou:

"A invenção desse jogo foi boa porque a gente se distrai com esse gatinho. Tem que fazê-lo entrar nesses becos e pegar os objetos, parece fácil, mas não é. Se apertar com força, ele anda demais, se apertar devagar, não é suficiente, ou seja, tem que achar o controle certo dos dedos para o gatinho não esbarrar nas paredes. Depois que a gente se adapta fica mais fácil e o jogo termina mais ligeiro. " (paciente CAPS)

Dada a importância do assunto, verificou-se que a utilização da ferramenta digital permitiu aos pacientes interagir espontaneamente enquanto executavam a aplicação. Essa liberdade foi o fator principal que os estimulou a participar ativamente das sessões terapêuticas computacionais.

\section{Conclusão}

Corroborando o que os pesquisadores citados nessa pesquisa revelaram em seus estudos, pôde-se observar in loco que a tecnologia, mais especificamente o uso da ferramenta Scratch, teve aplicabilidade real no centro terapêutico de saúde mental da cidade de Inhuma (Piauí). A ferramenta atuou como coadjuvante estimulador no processo terapêutico ocupacional, pois a aparente novidade despertou curiosidade, mas ao mesmo tempo medo, dado a peculiaridade do público alvo. Transtornos psíquicos aludem anseios de tristeza, inutilidade, dentre outros tantos que justificam a sensação de medo externada por alguns pacientes. Contudo, mesmo diante desses desafios, os resultados percebidos de forma observacional principalmente pelo Neuropsicopedagogo Adaljerry Ferreira de Sousa, contataram que a aplicação do software teve total relevância no que tange a terapia.

Apesar do receio retraído pelos pacientes por ser uma nova medida no tratamento, pôde-se constatar que a utilização da ferramenta aliada ao acompanhamento profissional mostrou eficiência e gerou motivação no exercício das atividades terapêuticas da instituição. Portanto, a utilização do Scratch no Centro de Atenção Psicossocial (CAPS) do município de Inhuma (Piauí) fez jus às expectativas criadas com a aplicação do experimento, atingindo assim o objetivo de incluir e socializar digitalmente aqueles pacientes.

\section{Trabalhos futuros}

Como trabalhos futuros recomenda-se a realização de pesquisas com o intuito de se descobrir novas ferramentas computacionais capazes de atuarem como coadjuvantes nas terapias desenvolvidas nos Centros de Atenção Psicossocial (CAPS). 
VIII Congresso Brasileiro de Informática na Educação (CBIE 2019)

Anais do XXV Workshop de Informática na Escola (WIE 2019)

\section{Referências}

Acioly, Y. (2009). Reforma psiquiátrica: Construção de outro lugar social para a loucura?. Paraná.

Bahri, M., Omar, H., Zaini, N., Norhazman, H., Mazalan, L., Latip, M., Taib, M., Ghazali, S. (2013). Depression Diagnostic and Screening Tools Using Android OS Platform.

Basaglia, F. (1982). Percepção dos agentes comunitários de saúde sobre as práticas de saúde mental na unidade básica de saúde da família do Paranoá no Distrito Federal. Distrito Federal.

Bastos, B., Borges, M., Abreu, J. (2010). Desenvolvimento de uma metodologia para o uso do Scratch for arduino no ensino médio. São Paulo.

Borycki, E., Keay. E. (2010). Methods to Assess the Safety of Health Information Systems.

Carey, M., Jones, K., Meadows, G., Sanson-Fisher, R., D’Este, C., Inder, K., Russell, G. (2014). Acuracy of general practitioner unassisted detection of depression.

Chattopadhyay, S. (2014). A neuro-fuzzy approach for the diagnosis of depression.

Cook, I., Balasubramani, G., Eng, H., Friedman, E., Young, E., Martin, J., Warden, D. (2006). Electronic source materials in clinical research: acceptability and validity symptom selfrating in major depressive disorder.

Drozd, F., Haga, S., Brendryen, H., Slinning, K. (2015). An Internet-Based Intervention (Mamma Mia) for Postpartum Depression: Mapping the Development from Theory to Practice.

Escribano, J. M., Montoya, B. (2012). Utilização do Software Scratch no Ensino das Ciências da Natureza e da Matemática com Alunos Portadores de Dislexia. Porto, Portugal.

Fortuna, T. R., Oliveira, M. R. (2011). Mapeamento das práticas comunicacionais radiofônicas como terapia psicossocial nos serviços de saúde mental no Brasil. Rio de Janeiro.

Franzoia, F., Pires, F., Teixeira, K., Lima, P. (2018). Programando para criar objetos de aprendizagem digitais de Ondulatória. InBrazilian Symposium on Computers in Education (Simpósio Brasileiro de Informática na Educação-SBIE) 2018 Oct 28 (Vol. 29, No. 1, p. 1063).

Gardner, W., Shear, K., Kelleher, K., Pajer, K.., Mammen, O., Buysse, D., Frank, E (2004). Computerized adaptive measurement of depression: A simulation study.

Graham, S. (2004). Novas tecnologias e inclusão digital. Salvador.

Marques, L. A. S. (2009). Avaliando a contribuição do Scratch para a aprendizagem pela solução de problemas e o desenvolvimento do pensamento criativo. Paraná.

Nunes, L., Magalhães, A., Madeira, S., Nunes, D., Nogueira, D., Passos, M., Macedo, E. (1998). Sistemas pictográficos de comunicação alternativa para portadores de paralisia cerebral. Brasília.

Oliveira, C. (2016). PARADEP - Ferramenta de apoio ao diagnóstico da depressão baseada na lógica para consistente. São Paulo.

Pinto, F. (2015). Utilização do Software Scratch no Ensino das Ciências da Natureza e da 
VIII Congresso Brasileiro de Informática na Educação (CBIE 2019)

Anais do XXV Workshop de Informática na Escola (WIE 2019)

Matemática com Alunos Portadores de Dislexia. Porto, Portugal.

Ribeiro, J. M., Dias, A. I. (2011). Políticas e inovação em atenção à saúde mental: limites ao descolamento do desempenho do SUS. Rio de Janeiro.

Ribeiro S, Melo A. (2017). Um Método para o Desenvolvimento de Software com Crianças Utilizando o Ambiente Scratch. InBrazilian Symposium on Computers in Education (Simpósio Brasileiro de Informática na Educação-SBIE) 2017 Oct 27 (Vol. 28, No. 1, p. 1027). 